\title{
Unsichere Erwerbsbeteiligung und Prekarität
}

Die Zunahme von Erwerbsformen, die vom Normalarbeitsverhältnis abweichen, verändert das deutsche Beschäftigungssystem. Der starken Nachfrage der Unternehmen nach Arbeitskraft, die ihnen externe Flexibilität der Beschäftigung ermöglicht, entspricht offenbar ein wachsendes Arbeitskraftangebot in diesen , atypischen “ Formen. Um zu unterscheiden, inwieweit diese Erwerbsformen für veränderte gesellschaftliche Teilhabemodi in der „Zone der Integration“ oder für unsichere Erwerbsmuster in der „Zone der Prekarität“ stehen, müssen Arbeitsmarktbeobachtung und Prekaritätsforschung den Haushaltszusammenhang und den Lebensverlauf berücksichtigen.

\section{Prekarität weiter denken - eine notwendige Kompli- kation}

„Prekarität“ steht in der politischen und wissenschaftlichen Debatte für zweierlei: für unsichere Formen der Erwerbsbeteiligung und für eine neue Erscheinungsform der „sozialen Frage“ in heutigen kapitalistischen Gesellschaften. Da die „Wiederkehr sozialer Unsicherheit" (Castel 2009, S. 21) vom Arbeitsmarkt und vom Beschäftigungssystem ausgeht, hängen engere und weitere Prekaritätsdiagnosen offenbar zusammen. Wie jedoch prekäre Positionen im Beschäftigungssystem und soziale Lagen miteinander vermittelt sind, wird selten genauer bestimmt. In diesem Beitrag wird vorgeschlagen, konzeptionell zwischen Erwerbsformen mit prekärem Potenzial, prekärer Arbeit im Haushaltszusammenhang und im Lebensverlauf und prekären sozialen Lagen zu unterscheiden. Dies erschwert zunächst die Aufgabe, klare Arbeitsbegriffe für die empirische Untersuchung zu entwickeln. Da man aber Prekarität nicht einfach mit atypischer Beschäftigung gleichsetzen kann, lässt sich diese Komplikation nicht vermeiden.

\subsection{WANN WIRD ERWERBSTEILHABE PREKÄR?}

Standen in den 1970er und 1980er Jahren zunächst die Ausgrenzungsrisiken, die mit Arbeitslosigkeit verbunden sind, im Mittelpunkt der Debatte, richtete sich die Aufmerksamkeit in der Folge auf Prekarisierung von Erwerbsarbeit. Die Zunahme atypischer Beschäftigungsverhältnisse passte nicht ins Bild eines einheitlich regulierten und gesicherten Lohnarbeitsverhältnisses, welches als „Normalar- beitsverhältnis“ (Mayer-Ahuja 2003) die „fordistische" Gesellschaftsentwicklung (Castel 2000, S. 286ff.) gekennzeichnet hatte. Diese Entwicklung setzt sich bislang ungebrochen fort. Nach Mikrozensus ist die Zahl der Erwerbstätigen in Deutschland von 1998 bis 2008 von 32,7 auf 34,7 Mio. gestiegen, doch die Zahl der in Normalarbeit $^{1}$ Beschäftigten ist von 23,7 Mio. auf 22,9 Mio., ihr Anteil von 72,6\% auf $66,0 \%$ zurückgegangen.

Je besser aber „atypische“ Erwerbsformen statistisch erfasst werden, desto unklarer wird, wann Erwerbsarbeit als prekär gelten sollte, wann also Erwerbsarbeit „subjektiv mit Sinnverlusten, Anerkennungsdefiziten und Planungsunsicherheit in einem Ausmaß verbunden ist, das gesellschaftliche Standards deutlich zuungunsten der Beschäftigten korrigiert" (Brinkmann et al. 2006, S. 17). ${ }^{2}$ Dies lediglich an Abweichungen vom Normalarbeitsverhältnis festzumachen, also schlicht mit der Zunahme atypischer Beschäftigung gleichzusetzen, erwies sich rasch als unzureichend (Evans/Gibb 2009, S. 20).

Das Normalarbeitsverhältnis (in der internationalen Debatte: standard employment relationship, SER) gibt keinen festen normativen Bezugspunkt ab; seine Merkmale sind von Land zu Land verschieden ${ }^{3}$ und längst selbst Gegenstand gesellschaftlicher Auseinandersetzung. So galt das deutsche Normalarbeitsverhältnis faktisch vor allem für männliche Familienernährer; die damit unterstellte geschlechtliche Arbeitsteilung von Erwerbs- und Sorgearbeit in Paarhaushalten ist mit einem Leitbild gleicher Wahlmöglichkeiten und Verwirklichungschancen von Männern und Frauen im Bildungs- und Beschäftigungssystem (Sachverständigenkommission 2011, S. 218) nicht vereinbar.

Die Konsequenz hieraus kann nur sein, prekäre Arbeit als Merkmal individueller Erwerbsverläufe oder Lebenslagen, nicht des
Beschäftigungsverhältnisses aufzufassen, und atypische Erwerbsformen demnach als „potenziell prekäre Beschäftigungsverhältnisse" (Mayer-Ahuja 2003, S. 54) anzusehen. Unter welchen weiteren Bedingungen aber machen sich diese Risiken geltend, wann also wird Arbeit prekär? So notwendig es war, das Prekaritätskonzept um persönliche, biografische und betriebliche Merkmale zu erweitern, die nicht dem Beschäftigungsverhältnis zuzuordnen waren (Rodgers 1989; Paugam 2000; ESOPE 2004; Brinkmann et al. 2006; Cranford/Vosko 2006), so viel schwieriger wurde die Verständigung auf einen Arbeitsbegriff und dessen statistische Operationalisierung.

Ausgangspunkt für die Untersuchung unsicherer Erwerbsarbeit könnte die Ver-

1 Als Normalarbeitsverhältnis definiert das Statistische Bundesamt vollzeitnahe Beschäftigung (mindestens 21 Wochenstunden) in unbefristetem Beschäftigungsverhältnis, mit Integration in die sozialen Sicherungssysteme und Identität von Arbeits- und Beschäftigungsverhältnis bei gleichzeitiger Weisungsgebundenheit des Arbeitnehmers an den Arbeitgeber (Wingerter 2009, S. 1081) Als atypisch gilt abhängige Beschäftigung in Teilzeit bis 20 Wochenstunden, in Minijobs, mit befristetem Arbeitsverhältnis und in Zeitarbeit; be den Selbstständigen werden Alleinselbstständige (ohne Beschäftigte) gesondert ausgewiesen

2 Das EU-Projekt ESOPE definiert "precarious employment " als Unterschreitung gesellschaftlich akzeptierter Normen, „which results from an unbalanced distribution (...) of the insecurity and risks typically attached to economic life in general and to the labour market in particular" (Frade et al., zitiert nach ESOPE 2004, S. 48).

3 "What is considered precarious in one country may be differently evaluated in another country" (ESOPE 2004, S. 45).

Peter Bartelheimer, Dr., Soziologe, vertritt den Forschungsschwerpunkt "Sozialmodell" des Soziologischen Forschungsinstituts (SOFI) an der Georg-August-Universität Göttingen e-mail: peter.bartelheimer@sofi.uni-goettingen.de 
ständigung darüber sein, wie Erwerbsarbeit Teilhabe vermittelt (Übersicht 1). Das Teilhabeversprechen, das im „Wohlfahrtsbzw. Teilhabekapitalismus" (Lutz 2007; Busch/Land 2011) historisch erstmals an relativ gesicherte Lohnarbeit geknüpft wurde, bezieht sich zugleich auf Beschäftigung und auf die Arbeitsverausgabung.

Die Teilhabeerwartungen, die sich an das Beschäftigungsverhältnis richten, können mit den vier Sicherheitsdimensionen beschrieben werden, die Wilthagen/Tros (2004) in ihrem „Flexicurity“Konzept den Flexibilitätsanforderungen gegenüberstellen: Arbeitsplatzsicherheit, Beschäftigungsstabilität, Einkommenssicherheit (was Ansprüche an die sozialen Sicherungssysteme einschließt) und Vereinbarkeitssicherheit, d.h. die Möglichkeit, Erwerbsarbeit und Sorgearbeit in der persönlichen Lebensführung kombinieren zu können. Obwohl die „vier Sicherheiten“ sich noch leicht den Regulierungsgegenständen des Normalarbeitsverhältnisses zuordnen lassen, bedingen sie bereits wesentliche Erweiterungen der Perspektive.

Arbeitskraft wird zwar individuell, aber von Erwerbspersonen in Haushalten angeboten. Das Arbeitskraftangebot wird daher wesentlich außerhalb des Betriebs, in Unterhalts- und Geschlechterarrangements auf Haushaltsebene und durch sozialstaatliche Regulierung geformt. Beschäftigungsverhältnisse gestalten nicht allein die Beziehung von Unternehmen zu ihren Arbeitskräften, sondern sie sind „Ergebnis von aufeinander bezogenen Auswahl- und Anpassungsprozessen in Betrieb und Privatsphäre" (Goedicke et al. 2007, S. 85ff.). Sie kommen in einem - aufgrund der ungleichen Marktmacht asymmetrischen - „doppelten Tausch“ (ebd.) zwischen Beschäftigten und Erwerbsorganisationen einerseits und Personen in Familien, Partnerschaften und privaten Netzwerken zustande, die insbesondere die Passung von Erwerbs- und Sorgearbeit betreffen.

Die wohlfahrtstaatliche Sicherung von Erwerbsarbeit sollte historisch erstmals auch den Lohnabhängigen eine langfristig planbare Lebensführung ermöglichen. Notwendig ist es daher, atypische Beschäftigung und ihre prekären Potenziale im individuellen erwerbsbiografischen Kontext zu bewerten (Kraemer 2008).

Auf der Grundlage der wohlfahrtsstaatlichen Sicherheiten, die an das Normalarbeitsverhältnis geknüpft wurden, entstand in der zweiten Hälfte des letzten Jahrhunderts ein historisch neuer Raum für subjektive Erwartungen an Arbeitsverhältnisse und Arbeitsbedingungen: Diese sollten die Fähigkeiten und Persönlichkeit der Arbeitenden entwickeln und ihnen Spielräume der Selbstverwirklichung eröffnen. Prekarität kann daher auch das Unterschreiten von Standards der Arbeitsinhalte und Arbeitsgestaltung, des Erhalts von Erwerbs- und Beschäftigungsfähigkeit und der Interessenvertretung am Arbeitsplatz bezeichnen.

Eine zusätzliche Erweiterung erfährt der Prekaritätsbegriff durch die Unterscheidung von Mustern subjektiver Verarbeitung flexibler und prekärer Arbeitsverhältnisse (etwa Dörre et al. 2006; Pelizzari 2009).

\subsection{ERWERBSTEILHABE MESSEN?}

Atypische und diskontinuierliche Beschäftigung wird also dann prekär, wenn sie die materielle Sicherung und den Gestaltungsspielraum der Lebensführung einschränkt, wenn sie die Arbeitenden von Mindeststandards guter Arbeit im Betriebszusammenhang ausschließt und wenn sie ihre subjektiven Erwerbsorientierungen verletzt. Für viele dieser Dimensionen gefährdeter Erwerbsteilhabe sind erst noch geeignete quantitative Datenzugänge

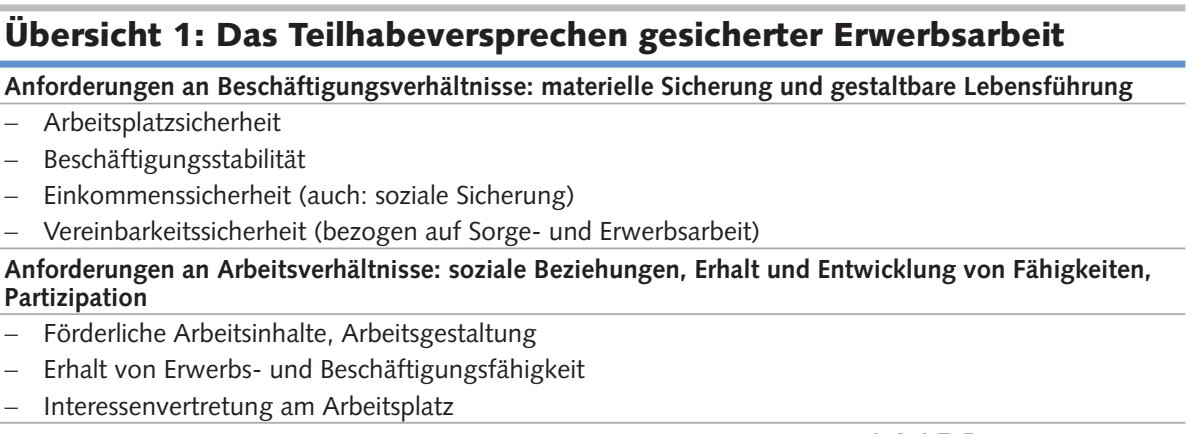
WSI MITTEILUNGEN und Beobachtungskonzepte zu entwickeln, wobei sich auch normative Fragen stellen.

Unsicherheit der Lebensführung bildet sich über die Zeit. Erwerbs- und Lebensverläufe begründen „Pfadabhängigkeiten“ und können prekäre Potenziale atypischer Beschäftigung „vererben“. Was aber kennzeichnet prekäre Erwerbsbeteiligung als biografisches Muster? Kommt es vor allem auf die Dauer eines Beschäftigungsverhältnisses an, das eine oder mehrere der genannten Teilhabenormen verletzt, oder auch auf die Zahl der Wechsel? Sind in verschiedenen Lebensphasen unterschiedliche Maßstäbe an Erwerbsteilhabe anzulegen? Welche Ressourcen sozialer Sicherung können Haushalte mobilisieren, um ihre Lebensführung bei unsicherer Erwerbsbeteiligung zu stabilisieren? Da in der Gesellschaft verschiedene Lebens- und Erwerbsmodelle von Paarhaushalten koexistieren und die Leitbilder der Gleichstellungs- und Familienpolitik inkonsistent und widersprüchlich sind (Sachverständigenkommission 2011, S. 217f.), fehlt für Vereinbarkeitssicherheit ein gesellschaftlich anerkannter Maßstab.

Der DGB-Index Gute Arbeit (2010) ${ }^{4}$ und die ILO-Initiative „Decent Work Agenda“ ${ }^{5}$ versuchen Normen für förderliche oder belastende Arbeitsbedingungen zu setzen und Indikatoren zu etablieren. Das Ergebnis ist aber zunächst eine größere Zahl von Einzelindikatoren. Summarische Aussagen über den Umfang prekärer Arbeit setzen einen Konsens über Aggregationsregeln voraus, der nicht besteht. So dürfte die Gesamtbewertung des DGB-Index (ebd.), wonach $15 \%$ „Guter Arbeit“ $52 \%$ „Mittelmäßiger Arbeit" und $33 \%$ „Schlechter Arbeit“ gegenüberstehen (ebd.), ${ }^{6}$ weniger konsensfähig sein als die Bewertung der Niveaus und Veränderungen bei einzelnen Indikatoren der drei Teilindizes Ressourcen, Belastungen sowie Einkommen und Sicherheit.

Beschäftigung und Arbeit können entgegengesetzte Vorzeichen annehmen: Als „unsichere Integration" bezeichnet Paugam (2000, S. 98ff.) die Situation von Beschäftigten, die trotz Beschäfti-

\footnotetext{
4 http://www.dgb-index-gute-arbeit.de/; Zugriff am 16.02.2011.

5 http://www.ilo.org/global/topics/decent-work/ lang--en/index.htm; Zugriff am 16.02.2011.

6 Der DGB-Index grenzt prekär und nicht-prekär Beschäftigte weiterhin über die Abweichung vom Normalarbeitsverhältnis ab und weist dann aus, wie unterschiedlich beide Gruppen ihre Arbeit bewerten.
} 
gungsunsicherheit die Möglichkeiten zur Selbstverwirklichung im Arbeitsvollzug hoch bewerten. Dagegen fällt beim Typus der „belastenden Integration“ stabile Beschäftigung mit einer wenig befriedigenden Arbeitssituation zusammen. Beide als gleichermaßen prekär zu bewerten, ginge mit einem erheblichen Informationsverlust einher.

Forschungspragmatisch empfiehlt es sich, den auch mit quantitativen Daten leichter beobachtbaren Merkmalen des Beschäftigungsverhältnisses schrittweise andere Informationen zuzuordnen (Cranford/Vosko 2006), die darüber entscheiden, wie weit das prekäre Potenzial atypischer Beschäftigung wirksam wird. Dies wird im Folgenden für den Haushaltszusammenhang und für die Lebensverlaufsperspektive skizziert.

\section{Der Haushaltszusammen- hang}

\subsection{ARMUTSRISIKO ATYPISCHER BESCHÄFTIGUNG}

Die abhängig Beschäftigten, die in atypischen Erwerbsformen arbeiten, sind zu gut einem Drittel (35,3\%) Alleinverdienende; jeder Zehnte von ihnen (9,4\%) lebt mit einer weiteren atypisch Beschäftigten zusammen (Wingerter 2009). In diesen beiden Konstellationen wird das Prekaritätsrisiko ungesicherter Erwerbsbeteiligung nicht durch gesicherte Beschäftigung eines anderen Haushaltsmitglieds ausgeglichen, entsprechend hoch ist mit 30,3\% bzw. 19,3\% das damit verbundene Armutsrisiko. ${ }^{7}$ Alleinlebende oder Alleinerziehende, die nur in Teilzeit oder geringfügig beschäftigt sind, leben zu zwei bis drei Fünfteln in relativer Armut.

Zur Hälfte jedoch leben die atypisch Beschäftigten in Haushalten mit wenigstens einem (meist männlichen) Normalarbeitnehmer. Gleiches gilt für ein Drittel der Soloselbstständigen. Drei Fünftel der Teilzeitbeschäftigten $(56,9 \%)$ und die Hälfte der Minijobber (49,9\%) - zumeist Frauen ${ }^{8}$ - ergänzen Haushaltseinkommen, zu denen ein Vollzeitbeschäftigter den größeren Teil beiträgt; ihr aktuelles Armutsrisiko ist daher gering.

Die Mehrzahl der zu Niedriglöhnen ${ }^{9}$ Beschäftigten lebt in Mehrverdienerhaus- halten und über der Armutsgrenze (Lohmann/Gießelmann 2010). Als potenziell prekär könnten Erwerbseinkommen definiert werden, die individuell nicht existenzsichernd wären, d.h. mit denen Alleinlebende die Interventionsschwelle der Grundsicherung unterschreiten würden.

\subsection{LEBENSFORMEN UND ERWERBS- KONSTELLATIONEN}

Dass sich Erwerbsformen mit prekärem Potenzial ausbreiten, kann nicht allein von der Nachfrageseite her, also durch betriebliche Personalstrategien und Arbeitsmarktbedingungen, erklärt werden. Die Ausbreitung von Erwerbsformen mit prekärem Potenzial geht auch auf - durch institutionelle Anreize mehr oder weniger unterstützte - Verdienermodelle und Geschlechterarrangements der Familienhaushalte zurück.

Im Jahr 2007 arbeiteten westdeutsche Männer im Erwerbsalter zu etwa zwei Dritteln (63,8\%), ostdeutsche Männer etwa zur Hälfte $(53,4 \%)$ im Normalarbeitsverhältnis $^{10}$ (Tabelle 1). Dagegen galt das Normalarbeitsverhältnis nur für gut ein Drittel (37,3\%) der westdeutschen Frauen im Erwerbsalter; zu jeweils etwa einem Viertel waren diese atypisch beschäftigt bzw. alleinselbstständig (28,7 \%) oder nicht erwerbstätig (25,5\%). Ostdeutsche Frauen waren annähernd zur Hälfte $(47,1 \%)$ in Normalarbeit und entsprechend seltener (zu 19,7\%) atypisch oder alleinselbstständig erwerbstätig; nur ein Sechstel von ihnen (15,5\%) stand außerhalb des Arbeitsmarkts.

Nur eine Minderheit der Familienväter ist atypisch beschäftigt bzw. alleinselbstständig: im Westen zwischen $10 \%$ und $15 \%$, im Osten zwischen $14 \%$ und $20 \%$. Frauen dagegen, die mit einem Partner und mit Kindern leben, sind im Westen eher selten (zu $17 \%$ bis $24 \%$ ) in Normalarbeit, jedoch zu zwei Dritteln nicht oder atypisch erwerbstätig. Obwohl die Frauenerwerbslosigkeit ${ }^{11}$ in den neuen Bundesländern mit $12,9 \%$ mehr als doppelt so hoch ist wie im Westen (5,6\%), sind ostdeutsche Frauen mit Partner und Kindern nur halb so häufig atypisch beschäftigt oder nichterwerbstätig wie westdeutsche Frauen der gleichen Lebensform. Ab dem dritten Lebensjahr der Kinder arbeiten ostdeutsche Mütter sogar häufiger in gesicherter Vollzeit als kinderlose Frauen. Neben dem Bedürfnis der Unternehmen nach externer
Flexibilität sind offenbar unterschiedliche Erwerbsorientierungen und Lebensformen der Haushalte als eigensinnige Motive für ein atypisches Arbeitsangebot zu berücksichtigen.

Die Verdienermodelle des Haushalts erweisen sich somit als wesentliche Kontextinformation bei der Bewertung atypischer Beschäftigung. In Westdeutschland realisiert nur eine Minderheit von 12,3\% der Paare mit Kindern das , adult worker model“ (Lewis 2004) partnerschaftlicher Erwerbsbeteiligung (Partnerin und Partner in annähernd gleichem Umfang erwerbstätig). In Ostdeutschland ist dies mit 37,7 \% das häufigste Verdienermodell für Elternpaare geblieben. In westdeutschen Familienhaushalten ist in der Regel nur ein Partner vollzeitnah erwerbstätig, die Partnerin (selten der Partner) verdient in Teilzeit oder Geringfügigkeit hinzu (45,6\% der Paare) oder bleibt ganz zu Hause (32,9 \% der Paare). ${ }^{12}$

Logistische Regressionen zeigen, dass die Lebensweise das Zustandekommen der

7 Nach EU-Standard liegt die Grenze relativer Einkommensarmut bei einem bedarfsgewichteten Haushaltseinkommen von weniger als $60 \%$ des Medians (Personengewichte nach neuer OECDSkala).

82008 arbeiteten 4,9 Mio. Beschäftigte bis zu 20 Stunden wöchentlich, 2,6 Mio. ausschließlich geringfügig. Von den erwerbstätigen Männern arbeiteten 3,4 \% bzw. $3 \%$ in diesen Erwerbsformen, von den erwerbstätigen Frauen 26,8 \% bzw. 12,6 \% (Janke/Wingerter 2010).

9 Üblicherweise wird die statistische Niedriglohnschwelle bei zwei Dritteln des mittleren Bruttostundenlohns (Median) angesetzt. Der übliche Schwellenwert relativer Einkommensarmut (vgl. Fn 7) und die Einkommensgrenzen für Ansprüche auf Mindestsicherungsleistungen beziehen sich auf das Haushaltsnettoeinkommen.

10 In Abgrenzung des Statistischen Bundesamts, vgl. Fn. 1.

11 Erwerbslosigkeit ist in Tabelle 1 nach der Definition des Statistischen Bundesamts und der Internationalen Arbeitsorganisation (ILO) abgegrenzt: Erwerbslos sind Personen ohne Erwerbstätigkeit, die sich in den letzten vier Wochen aktiv um eine Arbeitsstelle bemüht haben und sofort, d.h. innerhalb von zwei Wochen, für die Aufnahme einer Tätigkeit zur Verfügung stehen. Arbeitsuchende Nichterwerbspersonen sind nicht sofort verfügbar oder treten eine gefundene Stelle demnächst an. Die Arbeitslosmeldung spielt bei dieser Definition keine Rolle.

12 Daten des Mikrozensus 2007, Berechnung: Sabine Fromm, SOFI Göttingen. Sind Kinder unter dre Jahren zu versorgen, ist in Westdeutschland das traditionelle Alleinverdienermodell die häufigste Erwerbskonstellation (44,0 \% der Paare, in Ostdeutschland $32,9 \%$ ), bei Kindern im Schulalter überwiegt im Westen mit 50,7 \% das „moderni-

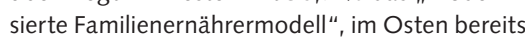
wieder das partnerschaftliche Modell. 
Tabelle 1: Erwerbsstatus nach Geschlecht und Haushaltstyp - in \% -

\begin{tabular}{|c|c|c|c|c|c|c|c|c|c|}
\hline \multicolumn{2}{|c|}{ Erwerbskonstellation } & \multirow{2}{*}{$\frac{1}{70,5}$} & \multirow{2}{*}{$\begin{array}{c}\mathbf{2} \\
10,1\end{array}$} & \multirow{2}{*}{$\begin{array}{c}3 \\
4,9\end{array}$} & \multirow{2}{*}{$\begin{array}{c}\mathbf{4} \\
5,7\end{array}$} & \multirow{2}{*}{$\begin{array}{c}5 \\
5,8\end{array}$} & \multirow{2}{*}{$\begin{array}{c}6 \\
0,8\end{array}$} & \multirow{2}{*}{$\begin{array}{c}7 \\
2,2\end{array}$} & \multirow{2}{*}{$\begin{array}{c}8 \\
100,0\end{array}$} \\
\hline Männer & Paar mit Kind unter 3 Jahren & & & & & & & & \\
\hline & Paar mit Kind zwischen 3 und $6 \mathrm{~J}$. & 73,4 & 8,0 & 5,0 & 6,5 & 5,0 & 0,7 & 1,4 & 100,0 \\
\hline & Paar mit Kind zwischen 7 und $12 \mathrm{~J}$. & 75,1 & 5,5 & 4,9 & 7,7 & 4,5 & 0,5 & 1,7 & 100,0 \\
\hline & Paar mit Kind zwischen 13 und $17 \mathrm{~J}$. & 74,4 & 5,1 & 4,7 & 7,6 & 4,4 & 0,7 & 3,0 & 100,0 \\
\hline & Paare ohne Kinder & 60,0 & 7,0 & 4,9 & 5,3 & 4,3 & 0,9 & 17,6 & 100,0 \\
\hline & sonstige Haushaltstypen & 64,6 & 9,1 & 3,7 & 5,1 & 6,8 & 1,0 & 9,6 & 100,0 \\
\hline & alleinerziehend & 54,6 & 11,4 & 4,2 & 3,6 & 13,4 & 2,4 & 10,5 & 100,0 \\
\hline & allein lebend & 57,4 & 10,6 & 6,0 & 3,2 & 10,8 & 2,0 & 9,9 & 100,0 \\
\hline & zusammen & 63,8 & 8,3 & 4,8 & 5,2 & 6,8 & 1,2 & 9,9 & 100,0 \\
\hline \multirow[t]{9}{*}{ Frauen } & Paar mit Kind unter $3 \mathrm{~J}$. & 24,3 & 20,6 & 2,6 & 0,8 & 2,8 & 2,8 & 46,0 & 100,0 \\
\hline & Paar mit Kind zwischen 3 und $6 \mathrm{~J}$. & 17,8 & 34,6 & 2,9 & 1,1 & 5,2 & 2,0 & 36,4 & 100,0 \\
\hline & Paar mit Kind zwischen 7 und $12 \mathrm{~J}$. & 17,0 & 40,9 & 4,2 & 2,0 & 4,6 & 1,0 & 30,3 & 100,0 \\
\hline & Paar mit Kind zwischen 13 und $17 \mathrm{~J}$. & 23,6 & 41,7 & 3,6 & 1,6 & 4,3 & 0,8 & 24,4 & 100,0 \\
\hline & Paare ohne Kinder & 43,3 & 17,4 & 2,9 & 1,6 & 4,2 & 0,8 & 29,9 & 100,0 \\
\hline & sonstige Haushaltstypen & 35,3 & 30,4 & 2,7 & 1,6 & 5,3 & 0,8 & 24,0 & 100,0 \\
\hline & alleinerziehend & 39,9 & 25,8 & 3,6 & 1,6 & 11,2 & 4,1 & 13,8 & 100,0 \\
\hline & allein lebend & 54,8 & 15,2 & 4,0 & 1,6 & 7,2 & 1,5 & 15,7 & 100,0 \\
\hline & zusammen & 37,3 & 25,5 & 3,3 & 1,6 & 5,6 & 1,4 & 25,5 & 100,0 \\
\hline \multicolumn{10}{|c|}{ Neue Bundesländer } \\
\hline \multirow[t]{9}{*}{ Männer } & Paar mit Kind unter $3 \mathrm{~J}$. & 61,2 & 13,8 & 6,9 & 3,4 & 10,3 & 1,7 & 2,6 & 100,0 \\
\hline & Paar mit Kind zwischen 3 und $6 \mathrm{~J}$. & 62,7 & 11,2 & 8,1 & 5,6 & 9,3 & 0,6 & 2,5 & 100,0 \\
\hline & Paar mit Kind zwischen 7 und $12 \mathrm{~J}$. & 65,9 & 7,4 & 7,4 & 6,5 & 9,2 & 1,4 & 2,3 & 100,0 \\
\hline & Paar mit Kind zwischen 13 und $17 \mathrm{~J}$. & 65,4 & 7,3 & 6,9 & 6,1 & 9,3 & 0,8 & 4,1 & 100,0 \\
\hline & Paare ohne Kinder & 49,6 & 9,5 & 4,9 & 4,7 & 10,1 & 1,3 & 20,0 & 100,0 \\
\hline & sonstige Haushaltstypen & 61,2 & 11,1 & 5,4 & 4,2 & 10,4 & 0,9 & 6,8 & 100,0 \\
\hline & alleinerziehend & 44,8 & 14,3 & 3,0 & 2,0 & 26,1 & 2,0 & 7,9 & 100,0 \\
\hline & allein lebend & 43,3 & 13,8 & 6,6 & 2,6 & 22,8 & 2,0 & 8,9 & 100,0 \\
\hline & zusammen & 53,4 & 11,1 & 5,8 & 4,1 & 14,0 & 1,4 & 10,3 & 100,0 \\
\hline \multirow[t]{10}{*}{ Frauen } & Paar mit Kind unter $3 \mathrm{~J}$. & 40,4 & 12,3 & 1,8 & 0,9 & 7,0 & 7,0 & 30,7 & 100,0 \\
\hline & Paar mit Kind zwischen 3 und $6 \mathrm{~J}$. & 49,1 & 16,8 & 4,3 & 1,9 & 9,9 & 3,1 & 14,9 & 100,0 \\
\hline & Paar mit Kind zwischen 7 und $12 \mathrm{~J}$. & 49,3 & 20,7 & 5,1 & 1,8 & 11,1 & 1,8 & 10,1 & 100,0 \\
\hline & Paar mit Kind zwischen 13 und $17 \mathrm{~J}$. & 54,9 & 17,1 & 4,9 & 2,0 & 12,2 & 2,0 & 6,9 & 100,0 \\
\hline & Paare ohne Kinder & 44,5 & 13,7 & 3,2 & 1,6 & 11,0 & 1,4 & 24,6 & 100,0 \\
\hline & sonstige Haushaltstypen & 54,8 & 16,8 & 3,3 & 2,3 & 11,7 & 1,2 & 9,9 & 100,0 \\
\hline & alleinerziehend & 41,4 & 17,9 & 3,4 & 1,5 & 20,5 & 4,3 & 11,1 & 100,0 \\
\hline & allein lebend & 42,5 & 17,3 & 4,1 & 1,5 & 15,6 & 2,0 & 16,9 & 100,0 \\
\hline & zusammen & 47,1 & 16,1 & 3,6 & 1,8 & 12,9 & 2,1 & 16,5 & 100,0 \\
\hline & & \multicolumn{4}{|c|}{$\begin{array}{l}\text { 1. Normalarbeit } \\
\text { 2. Atypische Beschäftigung } \\
\text { 3. Alleinstelbstständig } \\
\text { 4. Selbstständig mit Beschäftigung }\end{array}$} & \multicolumn{4}{|c|}{$\begin{array}{l}\text { 5. erwerbslos } \\
\text { 6. arbeitsuchende Nichterwerbspersonen } \\
\text { 7. sonstige Nichterwerbsarbeitpersonen } \\
\text { 8. Gesamt }\end{array}$} \\
\hline
\end{tabular}

Bevölkerung in Privathaushalten im Alter von 15 bis unter 65 Jahren, nicht in (Aus-)Bildung, ohne Wehr-, Zivildienstleistende. Abgrenzung von Normalarbeit wie in Fn. 1.

Quelle: Mikrozensus 2007 (SUF); Berechnung: Sabine Fromm.

WSI MITTEILUNGEN

verschiedenen atypischen Erwerbsformen unterschiedlich stark und in unterschiedlicher Richtung beeinflusst. Befristete Beschäftigung etwa wird vor allem nach vorausgegangenem Stellenwechsel wahrscheinlicher, nicht aber durch das Zusammenleben mit Kindern oder anderen Erwerbstätigen. Dagegen sind die beiden für Frauen charakteristischen Erwerbsformen kleiner Teilzeit und geringfügiger Beschäftigung ,untypisch für ostdeutsche Frauen [...] und werden sehr stark durch den Haushaltstyp beeinflusst" (Fromm 2010, S. 7). Obwohl vor allem Männer befristet oder bei Zeitarbeitsfirmen arbeiten, haben sie unter sonst gleichen Bedingungen auch bezogen auf diese Erwerbsformen „eine größere
Chance auf Beschäftigung in einem Normalarbeitsverhältnis als Frauen“ (ebd., S. 8).

\section{3}

\subsection{BEWERTUNGSFRAGEN UND MESSPROBLEME}

Um zu klären, ob unsichere Beschäftigung Teil eines auf Dauer prekären Musters der Erwerbsbeteiligung wird oder eine biografische Episode in einer Übergangssituation bleibt, müssen Sequenzmuster, d.h.
Abfolgen von Zuständen und Zustandswechseln, über längere Lebensphasen unterschieden werden.

Längsschnittdaten aus den Meldeverfahren der Sozialversicherungen (etwa die integrierten Erwerbsbiografien der Bundesagentur für Arbeit (BA) und des Instituts für Arbeitsmarkt- und Berufsforschung (IAB) oder die Versichertenbiografien der Rentenversicherung) ermöglichen die tagesgenaue Analyse individueller Erwerbsverläufe über lange Zeiträume und in hohen Fallzahlen (Alda/Bartelheimer 2008) und lassen sich zum Teil mit Betriebsdaten und Leistungsmerkmalen des sozialen Sicherungssystems verknüpfen, erfassen jedoch nur sozialversicherungs- 
Tabelle 2: Muster der Jugendphase, Geburtskohorten 1968-71 und 1973-76 - ausgewählte Anteilswerte in \% -

\begin{tabular}{|c|c|c|c|c|c|c|}
\hline Lebensverlaufsmuster & 1 & 2 & 3 & 4 & 5 & 6 \\
\hline Verteilung der Geburtskohorten auf Cluster & 18,3 & 20,2 & 12,1 & 15,2 & 24,2 & 9,9 \\
\hline Frauenanteil im Cluster (insgesamt: 52,9 \%) & 39,8 & 27,6 & 50,2 & 54,7 & 60,4 & 96,1 \\
\hline Anteil Elternschaft bis 30. Lebensjahr & 1,4 & 3,8 & 4,2 & 7,8 & 29,4 & 62,4 \\
\hline Anteil ohne Berufsabschluss bis 30 . Lebensjahr (insg.: 2,7 \%) & 2,4 & 3,3 & 0,6 & 1,8 & 2,4 & 6,9 \\
\hline \multicolumn{7}{|l|}{ Zeitanteile ... } \\
\hline im eigenen Haushalt & 16,0 & 9,8 & 53,3 & 38,3 & 48,1 & 65,2 \\
\hline in Ausbildung & 71,0 & 25,4 & 21,2 & 38,1 & 23,5 & 9,4 \\
\hline erwerbstätig & 21,8 & 69,1 & 72,5 & 50,6 & 47,0 & 28,0 \\
\hline - Anteil Erwerbszeit in Teilzeit (Frauen; insg.: 19,7 \%) & 30,8 & 3,8 & 12,2 & 4,7 & 17,4 & 43,6 \\
\hline - Anteil Erwerbszeit mit Befristung (insg.: 31,6 \%) & 51,6 & 18,1 & 21,7 & 26,0 & 28,8 & 41,0 \\
\hline arbeitslos & 2,6 & 1,4 & 3,7 & 6,8 & 3,7 & 5,7 \\
\hline in Erziehungszeit / nicht erwerbstätig & 0,2 & 0,6 & 0,5 & 1,5 & 11,1 & 34,7 \\
\hline \multicolumn{7}{|l|}{ Häufigkeit ausgewählter Wechsel } \\
\hline Auszug aus dem elterlichen Haushalt & 67,1 & 53,4 & 100,0 & 99,6 & 92,4 & 98,8 \\
\hline mehrfache Wechsel Ausbildung / Erwerbstätigkeit & 52,2 & 27,7 & / & 44,2 & 28,8 & / \\
\hline mind. ein Wechsel Ausbildung / Arbeitslosigkeit & $(21,0)$ & $(7,0)$ & / & / & 19 & I \\
\hline \multirow[t]{2}{*}{ mind. ein Wechsel Erwerbstätigkeit / Arbeitslosigkeit } & 17,6 & 25,0 & 53,6 & 33,9 & 39,3 & $(34,3)$ \\
\hline & \multicolumn{6}{|c|}{$\begin{array}{l}\text { 1. Lange Ausbildungszeit, späte Haushaltsgründung } \\
\text { 2. Arbeitsmarktintegration, späte Haushaltsgründungen } \\
\text { 3. Arbeitsmarktintegration mit Haushaltsgründung } \\
\text { 4. Ausbildung und Erwerbstätigkeit mit Haushaltsgründung } \\
\text { 5. Ausbildung, Erwerbstätigkeit, fühe Haushalts- u. Familiengründung } \\
\text { 6. Haushaltsgründung, Elternschaft, geringe Erwerbstätigkeit }\end{array}$} \\
\hline
\end{tabular}

pflichtige Beschäftigung vollständig und enthalten keine Haushaltsinformationen. Prospektive Panelerhebungen dagegen, etwa das Sozio-oekonomische Panel (SOEP) oder das Panel „Arbeitsmarkt und soziale Sicherung" (PASS) des IAB, lassen nicht jede Auswertung nach Regionen oder soziodemografischen Gruppen zu und bilden individuelle Beschäftigungszeiten nur monats- oder jahresgenau und für wenige Erwerbsformen ab, lassen sich dagegen insbesondere im SOEP mit anderen Ereignissen der persönlichen Lebensführung und mit Haushaltsinformationen verknüpfen. Typische Erwerbsverlaufsmuster können aber auch mit diesen Einschränkungen als Kontextinformation für Stichtagsinformationen zu atypischer Beschäftigung dienen. Die verbesserten Zugangsmöglichkeiten zu Mikrodaten ermöglichen es künftig, Zusatzwissen zum Betriebskontext mit Merkmalen individueller Erwerbsbeteiligung zu verknüpfen und Historien der Arbeitsverwaltung oder der Mindestsicherungssysteme in die Analyse prekärer biografischer Muster einzubeziehen.

\subsection{MUSTER DES ERWERBSVERLAUFS}

Im Folgenden werden exemplarisch Ergebnisse von Clusteranalysen erörtert, die mit Längsschnittdaten des SOEP für den Forschungsverbund Sozioökonomische Berichterstattung erstellt wurden (Schmidt
2011; Hacket 2011; Ebert/Trischler 2011). Individuelle Erwerbsverläufe wurden unter Berücksichtigung des Vorkommens, der Dauer und der Abfolge einzelner Zustände zu typischen Mustern gruppiert (Sequenzanalyse mittels „Optimal Matching“) und mit Daten der persönlichen Lebensführung verknüpft.

Bei zwischen 1968 und 1976 geborenen jungen Erwachsenen unterscheidet Schmidt (2011) monatsgenau für die Jugendphase von 17 bis unter 30 Jahren sechs Lebensverlaufsmuster (Tabelle 2). In den ersten beiden Gruppen überwiegen die jungen Männer: Sie bleiben lange im elterlichen Haushalt, und für sie haben offenbar Ausbildung und Arbeit Vorrang vor der Gründung einer Familie. Dabei befindet sich die erste Gruppe über 71,0 \% der Beobachtungszeit in Ausbildung; die Hälfte dieser Jugendlichen weist mehrfache Wechsel zwischen Ausbildung und Erwerbsarbeit auf. Die zweite Gruppe ist früher in den Arbeitsmarkt integriert: Beschäftigungszeiten füllen $69 \%$ der Jugendphase aus. Die dritte und vierte Gruppe junger Männer und Frauen verbindet Arbeitsmarktintegration mit Auszug bei den Eltern und Haushaltsgründung. Während die dritte Gruppe über drei Viertel (72,5 \%) der Jugendphase hinweg erwerbstätig ist, bleibt die vierte Gruppe etwas länger (38,1\% der Zeit) in Ausbildung. Im fünften Cluster stellen junge Frauen die Mehrheit, im sechsten sind sie unter sich. Ein Drittel (29,4\%) der fünften Gruppe und zwei Drittel $(62,4 \%)$ der sechsten Gruppe werden vor dem 30. Lebensjahr Eltern, Erziehungszeiten füllen $11,1 \%$ bzw. 34,7 \% ihrer Jugendphase aus. Von den familienorientierten jungen Frauen des sechsten Clusters haben 6,9\% keinen Berufsabschluss.

Hinweise auf prekäre Erwerbsmuster geben in dieser Analyse des Jugendalters vor allem die Zeitanteile in Befristung und die Erfahrungen mit Arbeitslosigkeit. Die jungen Erwachsenen des dritten Clusters werden gut zur Hälfte (53,6 \%) wenigstens einmal arbeitslos, aber offenbar nur für kürzere Zeiträume. Nur die des vierten und sechsten Clusters verbringen mit 6,8 \% bzw. 5,7 \% einen größeren Teil der Jugendzeit in Arbeitslosigkeit. Befristete Beschäftigung ist in allen Verlaufsclustern der Jugend hoch, konzentriert sich aber besonders im bildungsorientierten ersten Cluster und im sechsten Cluster.

Hacket (2011) typisiert Geburtskohorten von Erwerbspersonen in der Haupterwerbsphase (Altersspanne 26 bis unter 45 Jahre) nach deren monatsgenauem Erwerbsverlauf in Zeiträumen von fünf Jahren; dabei wird berücksichtigt, ob Personen mit Kindern im Haushalt zusammenleben (Tabelle 3). Auf die ersten drei, von Normalarbeit geprägten Erwerbsverläufe verteilen sich vier Fünftel der west- und ostdeutschen Männer mit 
Tabelle 3: Erwerbsverläufe in der Haupterwerbsphase - mit Kindern und ohne Kinder im Haushalt - ausgewählte Anteilswerte in \% -

\begin{tabular}{|c|c|c|c|c|c|c|c|}
\hline Erwerbsverlaufsmuster & 1 & 2 & 3 & 4 & 5 & 6 & 7 \\
\hline Verteilung der Erwerbspersonen auf Cluster & 23,9 & 5,4 & 30,1 & 11,2 & 6,9 & 11,9 & 10,7 \\
\hline Frauenanteil im Cluster & 18,2 & 26,7 & 38,1 & 98,2 & 66,3 & 64,5 & 99,9 \\
\hline Zeitanteil erwerbstätig & 98,1 & 95,7 & 94,5 & 86,9 & 69,3 & 44,8 & 13,1 \\
\hline Zeitanteil arbeitslos & 1,1 & 2,8 & 2,3 & 2,3 & 17,0 & 20,8 & 3,5 \\
\hline Zeitanteil in Mutterschaft & 0,4 & 0,6 & 1,1 & 10,5 & 6,9 & 12,7 & 82,7 \\
\hline ohne Kinder & - & - & 84,4 & - & - & 53,8 & - \\
\hline durchgängig mit Kind(ern) & 87,6 & $(6,0)$ & 7,4 & 86,6 & 76,2 & 15,9 & 87,3 \\
\hline mit wechselndem Familienstatus & 11,2 & 91,8 & 8,1 & 13,0 & 23,1 & 30,3 & 12,8 \\
\hline \multicolumn{8}{|c|}{ Verteilung der Erwerbspersonen nach Zeitraum, Region, Geschlecht } \\
\hline westdt. Männer 1991-95 & 39,7 & 8,2 & 37,2 & - & $(4,4)$ & 10 & - \\
\hline westdt. Männer 2001-05 & 37,8 & 7,8 & 42,2 & - & $(3,6)$ & 8,1 & - \\
\hline ostdt. Männer 1991-95 & 56,8 & $(5,8)$ & 28,5 & - & $(6,3)$ & - & - \\
\hline ostdt. Männer 2001-05 & 30,4 & $(11,4)$ & 37,2 & - & $(9,2)$ & $(11,9)$ & - \\
\hline westdt. Frauen 1991-95 & 6 & - & 22,2 & 19,7 & 6,6 & 13 & 30,7 \\
\hline westdt. Frauen 2001-05 & 3,6 & $(2,9)$ & 26,8 & 26,7 & 6,7 & 15,9 & 17,5 \\
\hline ostdt. Frauen 1991-95 & 37,1 & $(4,5)$ & $(9,7)$ & $(8,6)$ & 25,5 & $(9,5)$ & - \\
\hline ostdt. Frauen 2001-05 & 11,5 & $(5,9)$ & 16,7 & 20,2 & $(12,4)$ & 28,4 & - \\
\hline Zeitanteile in Erwerbsstatus ... & \multicolumn{7}{|c|}{ in Monaten } \\
\hline Vollzeit & 58,5 & 57 & 55,5 & 2,4 & 30,9 & 10,1 & 2,4 \\
\hline \multirow[t]{2}{*}{ Teilzeit } & 0,3 & 0,4 & 1,2 & 49,7 & 10,7 & 16,8 & 5,5 \\
\hline & \multicolumn{4}{|c|}{$\begin{array}{l}\text { 1. Normalerwerbsverlauf mit (Kindern) } \\
\text { 2. Normalerwerbsverlauf mit wechselndem } \\
\text { Familienstatus } \\
\text { 3. Normalerwerbsverlauf ohne Kinder } \\
\text { 4. Zuverdienstmodell }\end{array}$} & \multicolumn{3}{|c|}{$\begin{array}{l}\text { 5. diskontinuierliche, prekäre Verläufe mit } \\
\text { Kind(ern) } \\
\text { 6. diskontinuierliche, prekäre Verläufe, meist } \\
\text { ohne Kind(er) } \\
\text { 7. Familienarbeit }\end{array}$} \\
\hline \multicolumn{8}{|c|}{$\begin{array}{l}\text { Anmerkungen: Erwerbsverlauf über Fünf-Jahres-Zeiträume (1991-1995, 2001-2005). Berücksichtigt wird das Zusammenleben mit Kind(ern) im Haushalt, } \\
\text { unabhängig vom Verwandtschaftsverhältnis. } \\
\text { Quelle: Sozio-oekonomisches Panel; Hacket } 2011 \text { (Nummerierung der Cluster geändert). }\end{array}$} \\
\hline
\end{tabular}

Kindern (erste und zweite Gruppe) und ohne Kinder (dritte Gruppe). Frauen haben an diesem Muster nur dann nennenswert Anteil (38,1\% im dritten Cluster), wenn sie nicht mit Kindern zusammenleben. Typisch weibliche Muster sind das Zuverdienstmodell (viertes Cluster) und Familienarbeit (siebtes Cluster). Die Frauen in diesen beiden Gruppen leben im Beobachtungszeitraum genauso lange mit Kindern zusammen wie die Männer im ersten Normalarbeitscluster, sind aber fast gar nicht in gesicherter Vollzeit beschäftigt. Während die Zuverdienerinnen überwiegend in Teilzeit arbeiten, füllen bei den familienorientierten Frauen Mutterschaftszeiten den größten Teil des Fünfjahreszeitraums aus. Von besonderem Interesse für eine erweiterte Perspektive auf Prekarität sind die beiden „prekären“ Cluster fünf und sechs. Dort finden sich zu zwei Dritteln Frauen. In ihnen kommt es zu häufigen Wechseln zwischen Vollzeit, Teilzeit und Arbeitslosigkeit. Letztere nimmt im fünften Cluster 17,0\% der beobachteten Zeit ein, im sechsten Cluster 20,8\%.

Der Anteil der Männer, die nach einem der drei Vollzeitmuster arbeiten, blieb zwischen den beiden Beobachtungszeiträumen (1991 bis 1995, 2001 bis 2005) stabil.

\subsection{WAS DER LÄNGSSCHNITT BRINGT}

Typisierungen des Erwerbs- und Lebensverlaufs ermöglichen es, atypische Beschäftigung biografisch und sozialstrukturell besser zu verorten und zu berücksichtigen, dass Jugendalter, Haupterwerbsphase und höheres Erwerbsalter mit je eigenen Prekaritätsrisiken verbunden sind. In den Paneldaten lassen sich jedoch die erfassten Beschäftigungszeiten nur sehr grob nach ihrem Teilhabe- oder Prekaritätspotenzial differenzieren. Und es fehlt ein eigener Maßstab dafür, wann Erwerbsverläufe als prekär gelten sollen. Drei Befunde lassen sich vorläufig festhalten.

(1) Aus den Kohortenvergleichen, die hier nicht ausführlich dargestellt werden konnten (vgl. dazu Forschungsverbund 2011), ergibt sich, dass mit der Erosion des Normalarbeitsverhältnisses keine grundsätzlich neuen Muster des Erwerbsverlaufs entstanden sind. Der Umbruch im Beschäftigungssystem äußert sich vielmehr im wachsenden Gewicht diskontinuierlicher Verlaufsmuster, die in der Vergangenheit schwächer besetzt waren, etwa des Clusters mit langen Bildungszeiten und später Haushaltsgründung in der Jugendphase.
(2) Das Zusammenleben mit Kindern verändert die Erwerbsmuster von Männern und Frauen, aber in entgegengesetzter Richtung. Vaterschaft bestärkt Männer eher in ihrer Vollzeiterwerbstätigkeit, während Mutterschaft mit weniger intensiver und diskontinuierlicher Erwerbsbeteiligung einhergeht. In Ostdeutschland orientieren sich Paare mit Kindern weiterhin stärker an vollzeitnaher Erwerbsarbeit für beide Partner, den Frauen gelingt es jedoch seltener, dieses Modell zu realisieren.

(3) In allen Phasen der Erwerbsbiografie bestehen „fordistische“ Muster kontinuierlicher Vollzeiterwerbstätigkeit fort, während sich das Risiko von Arbeitslosigkeit oder atypischer Beschäftigung in bestimmten Verlaufsmustern konzentriert. ${ }^{13}$ Doch bietet kontinuierliche Vollzeitbeschäftigung keinen allgemein gültigen normativen Bewertungsmaßstab mehr: Nicht alle davon abweichenden, diskonti-

13 Das Fortbestehen vermeintlich "alter" Kontinuitätsmuster zeigt sich auch in den hier nicht dargestellten Altersübergängen (Ebert/Trischler 2011): In zwei von sechs Clustern gehen vorwiegend Männer aus weitgehend kontinuierlichen Vollzeiterwerbsverläufen direkt in den Ruhestand über. 
nuierlichen Muster können mit Prekarität gleichgesetzt werden.

\section{ENTSTEHT EINE ,ZONE DER PREKARITÄT"?}

Castel (2009, S. 28) bezweifelt mit guten Gründen, ob sich die „Wiederkehr der sozialen Unsicherheit“ zu einer „neuen gesellschaftlichen Klasse oder Unterklasse“ - dem Prekariat - verdichtet. In seinem „Zonenmodell“ (Castel 2000, S. 13 und 360f.), an dem sich ein großer Teil der Prekaritätsliteratur orientiert, entsprechen in der Zone der Integration einem stabilen Arbeitsverhältnis zugleich soziale Sicherungsansprüche und solide Eingliederung in soziale Beziehungen, wogegen für die Zone der Entkopplung das Fehlen produktiver Tätigkeit und soziale Isolation kennzeichnend sind. In der „instabilen Zwischenzone" der sozialen Verwundbarkeit fällt eine prekäre Stellung auf der „Achse der Integration durch Arbeit" mit weniger dichten Beziehungsnetzwerken etwa wie dem der Familie, zusammen. Und in der Zone der Fürsorge macht unsichere Erwerbsbeteiligung den Aufbau „sozialen Eigentums“ in Form von
Ansprüchen an die Sozialversicherung unmöglich.

Nur ein Teil der atypischen Beschäftigung dürfte in die Zone der Prekarität fallen. Dass Familien- und Sozialbeziehungen der unsicher Beschäftigten geschwächt sind, ist bei Castel nur eine Hypothese. Veränderte Lebensformen und rückläufige Anteile von Paarhaushalten sprechen noch nicht für ein abnehmendes Potenzial von Haushalten und Familien, Beschäftigungsrisiken zu kompensieren (vgl. Klenner et al. sowie Amacker in diesem Heft; Ebert/Fuchs 2011). Umgekehrt können Haushaltskonstellationen wie die der Familienernährerin Prekaritätsrisiken des Beschäftigtensystems verstärken (Klenner 2010). Dagegen überschneidet sich die Zone der Fürsorge zu großen Teilen mit jener der Prekarität. Das Sozialgesetzbuch Zweites Buch (SGB II) stützt als „Erwerbsfürsorge“ durch Zumutbarkeitsanforderungen und lohnergänzende Transfers ein wachsendes Arbeitskraftangebot im prekären Segment (Bartelheimer 2010).

Prekarität lässt sich auch als Bedeutungsverlust des „betriebszentrierten internen Arbeitsmarktsegments" (Lutz 2007) deuten, das in der zweiten Hälfte des letzten Jahrhunderts Teilhabe an der Wohlfahrtsentwicklung über Lohnarbeit vermittelte (Lutz 2007, S. 8; Bultemeier et al. 2008). Während dieses stabile Segment fortbesteht, weitet sich im Beschäftigungssystem ein externes, offenes und flexibles Arbeitsmarktsegment mit hohem prekärem Potenzial aus.

Zwar bleibt für einen großen Teil der atypisch Beschäftigten das Prekaritätsrisiko latent. Doch um die Veränderungen im Beschäftigungssystem bewerten zu können, muss die weitere Forschung Haushalte atypisch und diskontinuierlich Beschäftigter nach ihren ungleichen Möglichkeiten unterscheiden, die materiellen Risiken unsicherer Erwerbsbeteiligung zu kompensieren und Möglichkeiten und Bedingungen von Erwerbs- und Sorgearbeit zu wählen. Da sich gewählte Optionen und erzwungene Turbulenz des Lebensverlaufs in den gleichen Übergangsereignissen oder Verlaufsmustern äußern, wäre es notwendig, kontrafaktische Informationen über Erwerbswünsche und Lebensplanung in die Analyse einzubeziehen. Als prekär könnte dabei die Lage von Haushalten dann bezeichnet werden, wenn sie auf unsichere und nicht existenzsichernde Erwerbsformen mit instabilen Verläufen sowie auf nach Fürsorgelogik organisierte Sozialtransfers angewiesen sind, deren Bedingungen möglicherweise die Planbarkeit der Lebensführung weiter beeinträchtigen.

\section{LITERATUR}

Alda, H./Bartelheimer, P. (2008): Ungleiche Erwerbsbeteiligung Messkonzepte für ein segmentiertes Beschäftigungssystem, in: Gensior, S./Lappe, L./Mendius, H. G. (Hrsg.): Im Dickicht der Reformen Folgen und Nebenwirkungen für Arbeitsmarkt, Arbeitsverhältnis und Beruf, SAMF-Arbeitspapier (1), Cottbus, S. 49-78

Bartelheimer, P. (2010): Erwerbsfürsorge am segmentierten Arbeitsmarkt. Fünf Jahre Arbeitsmarkt- und Sozialpolitik nach dem SGB II, in: Mitteilungen aus dem SOFI 4 (10), November, S. 9-13

Brinkmann, U./Dörre, K./Röbenack, S. (2006): Prekäre Arbeit.

Ursachen, Ausmaß, soziale Folgen und subjektive Verarbeitungsformen unsicherer Beschäftigungsverhältnisse, Bonn

Bultemeier, A./Loudovici, K./Laskowski, N. (2008): Ist Prekarität überall? Unsicherheit im Zentrum der Arbeitsgesellschaft, in: Köhler, Ch./Struck, O./Grotheer, M./Krause, A./Krause, I./Schröder, T. (Hrsg.): Offene und geschlossene Beschäftigungssysteme. Determinanten, Risiken und Nebenwirkungen, Wiesbaden, S. 241-273

Busch, U./Land, R. (2011): Teilhabekapitalismus. Fordistische Wirtschaftsentwicklung und Umbruch in Deutschland 1950 bis 2009, in: Forschungsverbund Sozioökonomische Berichterstattung (im Erscheinen)
Castel, R. (2000): Die Metamorphosen der sozialen Frage. Eine Chronik der Lohnarbeit, Konstanz.

Castel, R. (2009): Die Wiederkehr der sozialen Unsicherheit, in: Castel, R./Dörre, K. (Hrsg.): Prekarität, Abstieg, Ausgrenzung. Die soziale Frage am Beginn des 21. Jahrhunderts, Frankfurt a.M., S. 21-34 Cranford, C. J./Vosko, L. F. (2006): Conceptualizing Precarious Employment: Mapping Wage Work across Social Location and Occupational Context, in: Vosko, L. F./Vosko, O. (Hrsg.): Precarious Employment. Understanding Labour Market Insecurity in Canada, Montreal, S. $43-66$

DGB-Index Gute Arbeit GmbH (2010): DGB-Index Gute Arbeit - Der Report 2010. Wie die Beschäftigten die Arbeitswelt in Deutschland beurteilen, Berlin

Dörre, K./Kraemer, K./Speidel, F. (2006): Prekäre Beschäftigung und soziale (Des-) Integration - Ursprünge, Konsequenzen und politische Verarbeitungsformen unsicherer Erwerbsarbeit, in: Forschungsinstitut Arbeit, Bildung, Partizipation (FIAB) (Hrsg.): Jahrbuch Arbeit, Bildung, Kultur 2005/06, Bd. 23/24, Recklinghausen, S. 9-40 
Ebert, A./Trischler, F. (2011): Altersübergänge, in: Forschungsverbund Sozioökonomische Berichterstattung (Hrsg.): Berichterstattung zur sozioökonomischen Entwicklung in Deutschland - Teilhabe im Umbruch, Zweiter Bericht, Wiesbaden (im Erscheinen)

Ebert, A./Fuchs, T. (2011): Haushalt, Familie und soziale Nahbeziehungen, in: Forschungsverbund Sozioökonomische Berichterstattung (Hrsg.): Berichterstattung zur sozioökonomischen Entwicklung in Deutschland - Teilhabe im Umbruch, Zweiter Bericht, Wiesbaden (im Erscheinen)

European Study on Precarious Employment (ESOPE) (2004): Precarious Employment in Europe: A Comparative Study of Labour Market related Risks in Flexible Economies, Luxembourg, http://ec.europa.eu/research/social-sciences/pdf/finalreport/ hpse-ct-2001-00075-final-report.pdf; (Stand 14.02.2011)

Evans, J./Gibb, E. (2009): Moving from Precarious Employment to Decent Work, Geneva

Forschungsverbund Sozioökonomische Berichterstattung (Hrsg.) (2011): Berichterstattung zur sozioökonomischen Entwicklung in Deutschland - Teilhabe im Umbruch, Zweiter Bericht, Wiesbaden (im Erscheinen)

Fromm, S. (2010): Wen trifft die Zunahme atypischer Beschäftigungsformen?, in: Mitteilungen aus dem SOFI 4 (10), November, S. 6-8

Fromm, S./Bartelheimer, P. (2011): Erwerbsteilhabe, in: Forschungsverbund Sozioökonomische Berichterstattung (Hrsg.): Berichterstattung zur sozioökonomischen Entwicklung in Deutschland - Teilhabe im Umbruch, Zweiter Bericht, Wiesbaden (im Erscheinen)

Goedicke, A./Diewald, M./Brose, H.-G. (2007): Ungleiche Partner gleicher Tausch? Zum Design einer Mehrebenenanalyse von sozialem Tausch in Beschäftigungsverhältnissen, in: Hummel, Hans J. (Hrsg.): Die Analyse von Gesellschaften, Organisationen und Individuen in ihrem Zusammenhang, Bonn, S. 77-104

Grunow, D./Kurz, K./Hillmert, S. (2005): Desintegration am Arbeitsmarkt? Eine empirische Analyse zur Stabilität von Erwerbsverläufen, in: Berger, J. (Hrsg.): Zerreißt das soziale Band? Beiträge zu einer aktuellen gesellschaftspolitischen Debatte, Frankfurt a.M./New York, S. $143-170$

Hacket, A. (2011): Erwerbsverläufe in der Haupterwerbsphase, in: Forschungsverbund Sozioökonomische Berichterstattung (Hrsg.): Berichterstattung zur sozioökonomischen Entwicklung in Deutschland Teilhabe im Umbruch, Zweiter Bericht, Wiesbaden (im Erscheinen) Janke, R./Wingerter, Ch. (2010): Atypische Beschäftigung - Strukturen und Folgen eines sich diversifizierenden Arbeitsmarktes, 3. soeb-Werkstattgespräch zur Erwerbsteilhabe 22./23. April, Göttingen, http:// www.soeb.de/img/content/werkstatt_2010_janke_wingerter.pdf
Klenner, Ch. (2010): Prekarisierungstendenzen im Lebenszusammenhang, Beitrag zum WSI-Herbstforum, 25.November, Berlin, http://www.boeckler.de/pdf/v_2010_10_25_klenner.pdf (letzter Zugriff am 4. März 2011)

Kraemer, K. (2008): Prekarität - was ist das?, in: Arbeit 17 (2), S. $77-90$

Lewis, J. (2004): Auf dem Weg zur "Zwei-Erwerbstätigen-Familie“, in: Leitner, S./Ostner, I./Schratzenstaller, M. (Hrsg.): Wohlfahrtsstaat und Geschlechterverhältnis im Umbruch, Wiesbaden, S. 62-84 Lohmann, H./Gießelmann, M. (2010): Armut von Erwerbstätigen in Ost- und Westdeutschland. Die Bedeutung von niedrigen Löhnen und unterschiedlichen Erwerbsmustern, in: Krause, P./Ostner, I. (Hrsg.): Leben in Ost- und Westdeutschland. Eine sozialwissenschaftliche Bilanz der deutschen Einheit 1990-2010, Frankfurt a.M., S. 299-311

Lutz, B. (2007): Wohlfahrtskapitalismus und die Ausbreitung und Verfestigung interner Arbeitsmärkte nach dem Zweiten Weltkrieg, Forschungsberichte aus dem Zentrum für Sozialforschung Halle e.V. (zsh) (4), Halle

Mayer-Ahuja, N. (2003): Wieder dienen lernen? Vom westdeutschen „Normalarbeitsverhältnis" zu prekärer Beschäftigung seit 1973, Berlin Paugam, S. (2000): Le salarié de la précarité. Les nouvelles formes de I'integration professionelle, Paris

Pelizzari, A. (2009): Dynamiken der Prekarisierung. Atypische Erwerbsverhältnisse und milieuspezifische Unsicherheitsbewältigung, Konstanz Rodgers, G. (1989): Precarious Work in Western Europe: The State of the Debate, in: Rodgers, G./Rodgers, J. (Hrsg.): Precarious Jobs in Labour Market Regulation. The Growth of Atypical Employment in Western Europe, International Institute for Labour Studies, Genf, S. 1-16 Sachverständigenkommission zur Erstellung des Ersten Gleichstellungsberichtes der Bundesregierung (2011): Neue Wege - gleiche Chancen. Gleichstellung von Frauen und Männern im Lebensverlauf. Gutachten der Sachverständigenkommission an das Bundesministerium für Familie, Senioren, Frauen und Jugend für den Ersten Gleichstellungsbericht der Bundesregierung, Essen/München

Schmidt, T. (2011): Junge Erwachsene, in: Forschungsverbund Sozioökonomische Berichterstattung (Hrsg.): Berichterstattung zur sozioökonomischen Entwicklung in Deutschland - Teilhabe im Umbruch, Zweiter Bericht, Wiesbaden (im Erscheinen)

Wilthagen, T./Tros, F. (2004): The Concept of "Flexicurity": A new Approach to Regulating Employment and Labour Markets, in: Transfer 10 (2), S. 166-186

Wingerter, Ch. (2009): Der Wandel der Erwerbsformen und seine Bedeutung für die Einkommenssituation Erwerbstätiger, in: Wirtschaft und Statistik (11), S. 1080-1098 